\title{
A Study of the Effects of Rotational Terms in the Power Transmission through Vibration Isolation Systems on Beam-like Structures
}

\author{
Ho-Jung Lee and Kwang-Joon Kim ${ }^{\dagger}$ \\ Center for Noise and Vibration Control, Dept. of Mechanical Engineering, KAIST, Science Town, Taejon, 305-701, Korea
}

(Received 18 January 2000; accepted 4 April 2000)

\begin{abstract}
The performance of a multi-dimensional vibration isolation system can be evaluated in several ways, one of which is to employ the concept of vibration power to define the isolation efficiency. It is especially useful in the high frequency range where the radiation of noise from the receiver structure is concerned. The idea is basically simple to understand but rather complicated to formulate and apply in practice. For an accurate estimation of power flow especially over a high frequency range, it is well known that, in principle, rotational motions should be taken into consideration together with translational motions at the isolator locations. In reality, however, power transmissions related to rotational terms are often neglected mainly due to difficulties in the instrumentation. In this paper, the effects of neglect of rotational terms in the power estimation are investigated for several different approximation methods; the mobility approach, impedance approach, and force and velocity approach. Since it is not possible to derive formulas analytically of the vibration power for structures of finite dimension, numerical simulations for a beam-like structure are presented to investigate the characteristics of the distortions in the power estimation induced by neglect of the rotational terms for each approximation approach.
\end{abstract}

${ }^{\dagger}$ Member of the International Institute of Acoustics and Vibration (IIAV)

\section{INTRODUCTION}

Various types of isolation techniques have been developed in the literature to reduce the vibration/force transmitted to receiver structures from excitation sources. Regarding the noise radiation in the high frequency range from the flexible receiver structures, a concept of power flow was employed. Although, mathematical formulations for the vibration power estimations have been almost completed, their application to practical systems still encounters difficulties, mainly due to instrumental limitations, especially with rotational motions. It is known that the contribution of the power transmission by the rotational terms to the total power should be taken into consideration as the frequency range of interest increases. Variability and complexity of the vibration isolation systems make it difficult to analyse the rotational power transmission through experimentation and to generalise its effects and, therefore, investigations made with numerical analysis for a simple beam-like source-isolator-receiver structure are presented in this study.

Goyder and White studied power flow analysis techniques for a mass-spring-receiver system with a single point connection where the receiver was an infinite beam or plate. They focused on the simplification of the practical structural analysis by using frequency response characteristics of an equivalent infinite structure for both force and torque excitations. ${ }^{1}$ Koh and White were concerned with the transmission of the vibration power into a beam- or plate-like machinery supporting structure at a single connection point which is subjected simultaneously to the action of sinusoidal force and moment excitations. ${ }^{2,3}$ They showed that the coupling mobility between the translational and the rotational terms can give rise to cancellation of the vibration power and also they illustrated how the vibration power can be minimised by adjusting the moment excitation.

Recent research by Swanson has showed that both overand under-estimation of the power transmission can be significant especially in the high frequency range if rotational stiffness of the isolator are not taken into account. ${ }^{4}$ Swanson's study involves a numerical simulation of a two-point mounted system with source and receiver consisting of freefree finite beam structures. ${ }^{4}$ Swanson has suggested that a non-dimensional ratio of the isolator's translational stiffness multiplied by the real part of the receiving structures' force mobility to its rotational stiffness multiplied by the one of moment mobility can be used as an index to determine whether the power transmission by the rotational motion is negligible or not. However, the relations of the erroneous peaks in the power approximations to the dynamic characteristics of the underlying system were not investigated in detail. Moorhouse and Gibbs have estimated the rotational power transmission for compressors in situ by employing two matched accelerometers at a given connection point. ${ }^{5}$ Petersson $^{6}$ and Yap and Gibbs ${ }^{7}$ have claimed that the moment becomes important even at low frequencies when translational motion is constrained in the proximity of a discontinuity. Yap and Gibbs estimated the rotational power transmission using transfer mobilities between excitation points and arbitrary points on the supporting structure. ${ }^{7}$ Howard and Hansen have employed vibration power as a cost function for active vibration isolation. ${ }^{8}$ They also showed, by finite element analysis, that there occur cancellations of power transmission due to the coupling between translational and rotational motions.

In the literature, the effect of rotational motions in vibration power estimation have been investigated for a sourceisolator-receiver system mostly with a single point connection either using numerical or experimental methods. The 